\title{
Serum cytokine levels are modulated by specific frequencies, amplitudes, and pulse widths of vagus nerve stimulation
}

Téa Tsaava ${ }^{1 *}$, Timir Datta-Chaudhuri ${ }^{2,3,4^{\star}}$, Meghan E. Addorisio ${ }^{1}$, Emily Battinelli Masi ${ }^{1,3}$, Harold A. Silverman ${ }^{1}$, Justin E. Newman ${ }^{1}$, Gavin H. Imperato ${ }^{1,4}$, Chad Bouton ${ }^{2,3,4}$, Kevin J. Tracey ${ }^{1,2,3,4, \mp}$, Sangeeta S. Chavan ${ }^{1,2,3,4, \mp}$, Eric H. Chang ${ }^{1,2,3, \ddagger}$

1. Laboratory of Biomedical Science, Feinstein Institutes for Medical Research, Northwell Health, 350 Community Drive, Manhasset, NY 11030, USA

2. Institute of Bioelectronic Medicine, Feinstein Institutes for Medical Research, Northwell Health, 350 Community Drive, Manhasset, NY 11030, USA

3. Donald and Barbara Zucker School of Medicine at Hofstra/Northwell, 500 Hofstra University, Hempstead, New York 11030, United States of America

4. The Elmezzi Graduate School of Molecular Medicine, 350 Community Drive, Manhasset, NY 11030, USA

${ }^{*}$ T.T. and T.D. are co-first authors.

‡ K.J.T, S.S.C., and E.H.C. are corresponding authors. K.J.T. (kjtracey@northwell.edu), S.S.C. (schavan@northwell.edi), E.H.C. (echang1@northwell.edu) 


\section{ABSTRACT}

Electrical stimulation of peripheral nerves is a widely used technique to treat a variety of conditions including chronic pain, motor impairment, headaches, and epilepsy. Nerve stimulation to achieve efficacious symptomatic relief depends on the proper selection of electrical stimulation parameters to recruit the appropriate fibers within a nerve. Recently, electrical stimulation of the vagus nerve has shown promise for controlling inflammation and clinical trials have demonstrated efficacy for the treatment of inflammatory disorders. This application of vagus nerve stimulation activates the inflammatory reflex, reducing levels of inflammatory cytokines during inflammation. Here, we wanted to test whether altering the parameters of electrical vagus nerve stimulation would change circulating cytokine levels of normal healthy animals in the absence of increased inflammation. To examine this, we systematically tested a set of electrical stimulation parameters and measured serum cytokine levels in healthy mice. Surprisingly, we found that specific combinations of pulse width, pulse amplitude, and frequency produced significant increases of the pro-inflammatory cytokine tumor necrosis factor alpha (TNFa), while other parameters selectively lowered serum TNFa levels, as compared to sham-stimulated mice. In addition, serum levels of the anti-inflammatory cytokine interleukin-10 (IL-10) were significantly increased by select parameters of electrical stimulation but remained unchanged with others. These results indicate that electrical stimulation parameter selection is critically important for the modulation of cytokines via the cervical vagus nerve and that specific cytokines can be increased by electrical stimulation in the absence of inflammation. As the next generation of bioelectronic therapies and devices are developed to capitalize on the neural regulation of inflammation, the selection of nerve stimulation parameters will be a critically important variable for achieving cytokine-specific changes. 


\section{INTRODUCTION}

Electrical stimulation is a fundamental neuromodulation technique used to activate nerves and muscles within the body. When electrical current is applied to nerve tissue using electrodes, there are many multiple variables that determine whether the desired neural fibers become activated. Generally, fiber activation by electrical stimulation follows a recruitment order from largest to smallest, with the smallest diameter fibers requiring the highest stimulation current levels (Baratta et al., 1989; Blair et al., 1933; Fang and Mortimer, 1991). Because different fiber types in the peripheral nervous system have varying functions and innervate different target organs, this fiber recruitment principle is important to achieve the desired physiological changes from activating nerves (Gorman and Mortimer, 1983). Electrical stimulation parameters such as output frequency, current, duration, and amplitude, are important determinants for achieving effective nerve activation that is both selective and efficient (Grill, 2015).

In the vagus nerve, there are three main fiber types: A-, B-, and C-fibers that can be distinguished on the basis of axon diameters, myelination, conduction velocity and stimulation thresholds for activation (Heck et al., 2002; Groves and Brown, 2005). This mixed nerve carries sensory afferent and motor efferent signals between the brain and the body to mediate vital functions of the autonomic nervous system (Bonaz et al., 2013; Tracey, 2002). A large body of preclinical studies and emerging clinical evidence indicates that electrical stimulation at the cervical vagus nerve is able to change the body's immune response to injury or infection, specifically by reducing the levels of certain serum cytokines that are important mediators of inflammation in the body (Andersson and Tracey, 2012; Borovikova et al., 2000). This stimulation of the vagus nerve regulates cytokine release from the spleen through activation of the inflammatory reflex, thereby protecting against lethality in models of systemic inflammation (Borovikova et al., 2000; Chavan et al., 2017; Tracey, 2002). Early clinical evidence for the therapeutic efficacy of this electrical vagus nerve stimulation has shown promise to treat patients with chronic inflammatory disorders (Bonaz et al., 2016; Koopman et al., 2016). This body of evidence has also demonstrated that electrical stimulation parameters can be modified to intentionally elicit different physiological effects, such as the separation of anti-inflammatory and cardioinhibitory effects (Huston et al., 2007). The anti-inflammatory effects of vagus nerve stimulation have been attributed to $A$ - and B-fiber activation, while the cardioinhibitory effects are thought to be mediated by only B-fibers (Huston et al., 2007; Olofsson et al., 2015; Yoo et al., 2016). 
As differential fiber recruitment is affected by nerve stimulation parameters, we wondered how specific stimulation parameters might effect serum cytokine levels in a parameter-dependent fashion. To accomplish this, we modified the output of our electrical stimulator to select frequencies, amplitudes, and pulse widths predicted to differentially activate different classes of fibers within the vagus nerve. Here we observed that certain cytokines, such as tumor necrosis factor alpha (TNFa) and interleukin 10 (IL-10), could be increased or decreased by specific combinations of frequency, pulse width, and pulse amplitudes in healthy mice.

\section{METHODS}

Animals

Naïve male BALB/c mice (8 to 12 weeks old) were obtained from Charles River Laboratories (Wilmington, MA, USA) and acclimated for at least one week before conducting experiments. Animals were housed on a $12: 12$ hour reverse light/dark cycle at $23^{\circ} \mathrm{C}$ and relative humidity 30 $70 \%$. Standard chow was withheld from animals for a period of up to three hours prior to stimulation of the cervical vagus nerve. Water was available ad libitum. All experiments were performed under protocols approved by the Institutional Animal Care and Use Committee of the Feinstein Institutes for Medical Research and in strict adherence with NIH guidelines on the care and use of laboratory animals.

\section{Vagus nerve isolation and electrical stimulation}

All surgical procedures were conducted using aseptic technique. Mice were administered isoflurane anesthesia through a nose cone in the supine position (oxygen flow $1 \mathrm{~L} / \mathrm{min}$, isoflurane $1.75 \%)$. An appropriate depth of anesthesia was assessed by toe pinch reflex. The cervical region was shaved with an electric razor and then sterilized with $70 \%$ ethanol. A midline incision was made, the salivary glands were identified and bluntly dissected to expose the left carotid bundle lateral to the sternocleidomastoid muscle. The left cervical vagus nerve was separated from the carotid sheath and placed on a $200 \mu \mathrm{m}$ diameter micro cuff sling bipolar electrode with platinumiridium contacts (CorTec $\mathrm{GmbH}$, Freiburg, Germany). Parafilm was placed over the surgical site to prevent desiccation of the nerve during stimulation. Heart rate was continuously monitored using a MouseSTAT® Heart Rate Monitor (Kent Scientific, Torrington, CT, USA).

Electrical pulses were delivered by a constant current stimulator system PlexStim 2.0 (Plexon, Dallas, TX) and individually controlled with PlexStim v2.2 software. The following stimulation

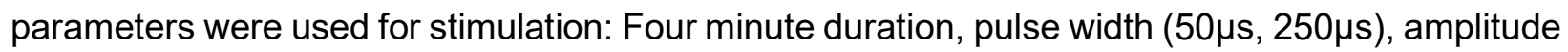
$(50 \mu \mathrm{a}, 200 \mu \mathrm{A}$, and $750 \mu \mathrm{A})$, and frequency $(30 \mathrm{~Hz}, 100 \mathrm{~Hz})$. Sham operated mice underwent the 
same surgical procedures but without electrical stimulation. After stimulation, the skin was sutured closed and the animals were returned to their home cages for recovery.

\section{Serum collection and analysis}

Two hours following stimulation, whole blood was collected from animals by cardiac puncture following euthanasia by $\mathrm{CO}_{2}$ asphyxiation. Blood was allowed to clot in a polypropylene tube at room temperature for 30-60 min. To obtain serum, the tubes were centrifuged two times, first at $5000 \times \mathrm{g}$ for 10 minutes, followed by $10000 \times \mathrm{g}$ for 2 minutes. The supernatant serum was collected in a clean tube and stored at $-20^{\circ} \mathrm{C}$ until further processing. Serum was analyzed on multiplex cytokine immunoassay plates (V-PLEX Panel 1 mouse kit; Meso Scale Discovery, Rockville MD) to quantify levels of IFN-y, IL-1ß, IL-2, IL-4, IL-5, IL-6, CXCL1, IL-10, IL-12p70, and TNF- $\alpha$.

\section{Statistical analysis}

Differences in serum cytokine levels between stimulated and non-stimulated (sham) groups were analyzed by Mann-Whitney $U$ tests (Prism 8.0). In all tests, $P<0.05$ was accepted as an indication of statistical significance.

\section{RESULTS}

Here we utilized an established methodology to surgically isolate and electrically stimulate the vagus nerve. For all experiments, stimulation was performed on experimental animals with shammatched controls. The vagus nerve at the cervical level was surgically isolated and a cuff electrode was placed on the nerve. Following stimulation, the mouse was recovered for two hours, then euthanized for tissue collection (Figure 1A). During electrical stimulation, depolarization of the nerve was achieved via cathodal current application at the electrode-tissue interface and at the electrode with the lower electrical potential. We utilized biphasic waveforms, with a secondary anodic phase to balance out the net effect charge to zero (Figure 1B). Without charge balancing, a detrimental electrical potential buildup at the electrode may occur over time resulting in both tissue damage and reduction of electrode effectiveness. The stimulation waveforms for the different parameters are shown in Figure 1B.

While electrical stimulation is known to decrease serum TNFa in a setting of increased inflammation, its effect during normal physiology is unclear. To assess this, we delivered cervical 
stimulation to normal mice and measured serum cytokines two hours later. We found that specific combinations of stimulation parameters significantly changed levels of serum TNFa. Specifically, stimulation at the short pulse width $(50 \mu \mathrm{s})$ at $30 \mathrm{~Hz}$ pulse and $200 \mu \mathrm{A}$ amplitude produced a significant decrease in TNFa (Mann Whitney $U=216, P<0.05$; Figure 2A). Stimulation with a 50 $\mu$ s pulse width at $100 \mathrm{~Hz}$ and $750 \mu \mathrm{A}$ also resulted in a decrease in serum TNFa (Mann Whitney $\mathrm{U}=6, P<0.05$; Figure 2B). When we increased the pulse width to $250 \mu$ s during stimulation, we observed a significant increase in serum TNFa levels at $30 \mathrm{~Hz}$ and $750 \mu \mathrm{A}$, compared to sham mice (Mann Whitney test, $U=60, P<0.0001$; Figure 3A). In contrast, stimulation $250 \mu$ s and 100 $\mathrm{Hz}$ produced no statistically significant changes in serum TNFa (Mann Whitney $\mathrm{U}=38, P=0.17$; Figure 3B). These results suggest that specific stimulation parameters can alter serum TNFa in a bidirectional manner.

IL-10 is a potent anti-inflammatory cytokine that suppresses Th1 cells, NK cells, and macrophages during infection (Couper et al., 2008; Hutchins et al., 2013). To examine whether electrical stimulation could also change levels of serum IL-10, we used specific combinations of frequency, pulse width, and amplitude followed by serum IL-10 measurements. Interestingly, the shorter $50 \mu$ s pulse width at $30 \mathrm{~Hz}$ produced statistically significant increases in serum IL-10 at both the $50 \mu \mathrm{A}$ and $750 \mu \mathrm{A}$ amplitudes $(50 \mu \mathrm{A}$, Mann Whitney $U=115, P<0.05 ; 750 \mu \mathrm{A}$, Mann Whitney $\mathrm{U}=53, P<0.05$; Figure 4A). Meanwhile, a $50 \mu$ s pulse width at $100 \mathrm{~Hz}$ stimulation resulted in no significant changes in IL-10 levels (Mann Whitney $U=34, P=0.96$; Figure 4B). With the longer $250 \mu$ s pulse width, we observed statistically significant increases in IL-10 at $750 \mu \mathrm{A}$ amplitude for both the $30 \mathrm{~Hz}$ (Mann Whitney $U=53, P<0.05$; Figure 5A) and $100 \mathrm{~Hz}$ frequencies (Mann Whitney $\mathrm{U}=18, P<0.001$; Figure 5B). There was also a significant increase in IL-10 at 100 $\mathrm{Hz}$ with the $250 \mu$ s pulse width at $50 \mu \mathrm{A}$ amplitude (Mann Whitney $\mathrm{U}=20, P<0.05$; Figure 5B). This demonstrates that specific parameters have a different effect on serum IL-10, compared to TNFa.

Electrical nerve stimulation-induced bradycardia has been used to index nerve fiber activation during stimulation, specifically activation of the intermediate diameter B-fibers within the vagus nerve (Musselman et al., 2019; Yoo et al., 2013; Yoo et al., 2016). During our stimulation experiments, we measured heart rate with a continuous heart rate monitor to obtain an indirect measure of B-fiber recruitment. Stimulation with $50 \mu \mathrm{s}$ pulses resulted in bradycardia only at the $750 \mu \mathrm{A}$ and $30 \mathrm{~Hz}$ parameter $(-17.1 \pm 2.9 \%$ decrease; Figure $6 \mathrm{~A})$. The $50 \mu$ sulse width stimulation did not produce bradycardia at $100 \mathrm{~Hz}$ for any of the tested pulse widths (Figure 6B). With the longer $250 \mu$ s pulse at $30 \mathrm{~Hz}$, bradycardia was produced at both $200 \mu \mathrm{A}$ and 750 
$\mu \mathrm{A}$ pulse amplitudes (Figure 6C). At $100 \mathrm{~Hz}$, stimulation with $250 \mu \mathrm{s}$ pulse width produced the largest measured decrease in heart rate (-20.4 $\pm 4.4 \%$; Figure 6D). These results indicate that alteration of stimulation parameters results in differential effects on heart rate, with longer pulse width increasing the likelihood of recruiting cardiac innervating B-fibers in the mouse vagus nerve.

In addition to assessing changes in the prototypical pro-inflammatory cytokine TNF $\alpha$ and anti-inflammatory cytokine IL-10, we also measured the serum levels for a set of additional cytokines including: interferon gamma (IFN-ץ), interleukin-12 p70 (IL-12 p70), interleukin-1 beta (IL-1ß), interleukin-2 (IL-2), interleukin-4, (IL-4), interleukin-5 (IL-5), interleukin-6 (IL-6), and chemokine ligand C-X-C motif ligand 1 (CXCL1). We found that the serum levels of certain proinflammatory cytokines, such as IL-6, were increased across a wide range of stimulation parameters for both the short $50 \mu$ s and long $250 \mu$ s pulse widths (Table 1, Table 2). Serum CXCL1 was also significantly increased by stimulation across several parameter combinations. For anti-inflammatory cytokines, we did not observe changes in IL-4 for any stimulation parameter combination. These results suggest that certain cytokines are more susceptible to modulation via electrical stimulation than others. It should be noted that the measured concentrations of IFNy, IL-1 $\beta$, IL-2, IL-4, and IL-5 were very low across the sham and stimulation groups such that, while there may be statistically significant changes with some of the stimulation parameters, the low concentrations suggest a lack of physiological relevance for these specific cytokines (Table 1, Table 2).

\section{DISCUSSION}

The neural control of immunity through stimulation of the vagus nerve holds significant promise for treating inflammatory disorders. Here we have shown that a set of electrical stimulation parameters can change specific cytokine levels in the absence of inflammation. We found, to our surprise, that electrical stimulation of the vagus nerve with specific parameters in conditions of low circulating cytokines (i.e. healthy control mice) results in an elevation in serum levels of TNFa and IL-10, cytokines known to be involved in inflammatory responses. These results indicate that systemic cytokines respond to specific combinations of frequency, amplitude, and pulse width applied to the cervical vagus nerve. How these parameters affect the recruitment of specific fiber types within the vagus nerve remains a topic of important ongoing work, but our results indicate that the refinement of these parameters may be important in the neuromodulation of immunological responses mediated through vagal signaling. 
The effect of vagus nerve stimulation on cytokines in the absence of systemic inflammation has not, to our knowledge, been carefully examined. This is the first demonstration that specific stimulation parameters can be used to increase serum TNFa and IL-10. TNFa is an inflammatory cytokine that is released rapidly by macrophages following infection and injury. Transient elevations in serum TNFa are required to coordinate host defense against pathogens and the repair of injured host tissue. While physiological inflammation is locally protective, pathological inflammation caused by persistent and unregulated TNF levels cause chronic tissue damage and drive the pathogenesis of inflammatory disease including rheumatoid arthritis, Crohn's disease, and inflammatory bowel disease (Bonaz et al., 2016; Kalliolias 2016; Koopman et al., 2016). A large body of work established the critical role of TNFa in the pathogenesis of systemic inflammation in animal models and led to the clinical translation of TNFa blockade to treat chronic inflammation. The beneficial effect of vagus nerve stimulation for reducing TNFa in the context of inflammatory disorders is clear, however, an increase in proinflammatory cytokine levels may also be beneficial in certain physiological and pathologic contexts. An increase in circulating cytokines might be useful to bolster the immune system in conditions of immunosuppression, for example, due to immunodeficiencies or viral infection (Breen, 2002; Varzaneh et al., 2014). There is also evidence that certain cancer patients have lowered levels of certain circulating cytokines that might benefit from immunotherapy to increase those levels (Khan et al., 2018). As techniques are developed to better understand the relationship between vagus nerve activity and cytokine signaling (Steinberg et al., 2016; Tsaava et al., 2019; Zanos et al., 2018), there may also be other physiological conditions that would benefit from intentionally increasing cytokine levels.

Our results suggest that serum cytokines may likewise be controlled by specific fiber sets and firing patterns. Altering the parameters of the stimulation pulse affects the specific vagus nerve fibers that are recruited. A large body of evidence shows that increasing pulse width and amplitude, and consequently the charge delivered during the both the cathodic and anodic phases of the pulse, inhibits activation of large diameter fibers while selectively activating smaller diameter fibers (Baratta et al., 1989; Musselman et al., 2019). For example, lower amplitude stimulation activates A-fibers but as stimulation increases, the A-fiber activation becomes suppressed and B-fibers become activated to change heart rate (Burke et al., 1975). It is possible that the differential effect on heart rate may be a result of effective recruitment of large and small fiber types with the long pulse at lower amplitudes, and selective inhibition of larger fibers at the larger pulse widths. The effective pulse widths and stimulus amplitudes necessary to elicit heart rate changes is shown in Figure 6. As the stimulation charge increased, we generally observed 
more pronounced bradycardia, however, heart rate decreases were not always coupled to changes in serum cytokines. The largest increase in serum TNFa at $250 \mu$ s pulse width, $30 \mathrm{~Hz}$, and $750 \mu \mathrm{A}$ was associated with the largest decrease in heart rate, but TNFa levels did not change for two other stimulation parameters that induced bradycardia (Figure 6: $50 \mu$ sulse width, 30 $\mathrm{Hz}, 750 \mu \mathrm{A}$ and $250 \mu \mathrm{s}$ pulse width, $100 \mathrm{~Hz}, 750 \mu \mathrm{A})$. This dissociation between bradycardia and serum cytokines levels confirms prior work indicating that cytokine effects are independent of the B-fiber activation that induces bradycardia (Huston et al., 2007).

We have considered the possibility that different durations of total stimulation may also be an important variable in cytokine-specific modulation via the vagus nerve. Further, while the applicability of these findings may be most relevant for disease conditions, these studies were carried out in healthy control animals. Therefore, they should be interpreted with caution if trying to extrapolate to specific disease conditions. The parameter space of electrical nerve stimulation is important because varying timing, duration, and amplitude should affect activation of different target organs and brain structures. Electrical stimulation delivered to cervical vagus nerve is broadly applied to all nerve fibers, including sensory afferents and motor efferents. It is thought that B-fibers mediate motor efferent control of the visceral organs. Because the vagus nerve innervates several major organs in the viscera, stimulation of this neve has the potential to treat a broad range of disorders ranging including but not limited to arthritis, myocardial infarctions, and metabolic disorders (Kong et al., 2012; Levine et al., 2014; Chang et al., 2019).

The unexpected results here indicate that it is possible to stimulate the production of TNFa and other cytokines using electrical stimulation of the cervical vagus nerve. As bioelectronic therapies continue to evolve for controlling inflammation, increasingly refined stimulation strategies will be needed to activate fibers of interest (Grill, 2015; Tsaava et al., 2019), while also minimizing the potential for the negative outcomes and off-target effects associated with vagus nerve stimulation. The effectiveness of these selective stimulation paradigms will depend on knowing which specific fiber populations mediate the neural regulation of inflammation and the optimal techniques for activating them. Our results show that varying stimulation parameters of frequency, amplitude, and pulse width holds promise for the specific regulation of cytokine responses in both health and disease. 


\section{REFERENCES}

Andersson, U., Tracey, K.J. (2012) Neural reflexes in inflammation and immunity. J. Exp. Med. 209: 1057-68.

Baratta, R., Ichie, I., Hwang, S.K., Solomonow, M. (1989) Orderly stimulation of skeletal muscle motor units with tripolar nerve cuff electrode. IEEE Transactions on Biomedical Engineering 36(8): 836-843.

Blair, E. A., Erlanger, J. (1933) A comparison of the characteristics of axons through their individual electrical responses. American Journal of Physiology-Legacy Content 106.3: 524-564.

Bonaz, B., Sinniger, V., Hoffmann, D., Clarençon, D., Mathieu, N., Dantzer, C., Vercueil, L., Picq, C., Trocmé, C., Faure, P., Cracowski, J.-L., Pellissier, S. (2016) Chronic vagus nerve stimulation in Crohn's disease: a 6-month follow-up pilot study. Neurogastroenterol. Motil. 28(6):948-53. doi: 10.1111/nmo.12792.

Bonaz, B., Picq, C., Sinniger, V., Mayol, J.F., Clarencon, D. (2013) Vagus nerve stimulation: from epilepsy to the cholinergic anti-inflammatory pathway. Neurogastroenterol Motil 25(3): 208-21. doi: 10.1111/nmo.12076.

Borovikova, L.V., Ivanova, S., Zhang, M., Yang, H., Botchkina, G.I., Watkins, L.R., Wang, H., Abumrad, N., Eaton, J.W., Tracey, K.J. (2000). Vagus nerve stimulation attenuates the systemic inflammatory response to endotoxin. Nature 405(6785):458-62.

Breen, E.C. (2002) Pro- and anti-inflammatory cytokines in human immunodeficiency virus infection and acquired immunodeficiency syndrome. Pharmacol Ther 95(3): 295-304.

Burke, D., Mackenzie, R.A., Skuse, N.F., Lethlean, A.K. (1975) Cutaneous afferent activity in median and radial nerve fascicles: a microelectrode study. J Neurol Neurosurg Psychiatry 38(9):855-64.

Chavan, S. S., V. A. Pavlov, K. J. Tracey. (2017) Mechanisms and Therapeutic Relevance of Neuro-immune Communication. Immunity 46: 927-942.

Chang, E.H., Chavan, S.S., Pavlov, V.A. (2019) Cholinergic control of inflammation, metabolic dysfunction, and cognitive impairment in obesity-associated disorders: mechanisms and novel therapeutic opportunities. Front Neurosci 13: 263.

Couper, K.N., Blount, D.G., Riley, E.M. (2008) IL-10: The master regulator of immunity to infection. J Immunol 180: 5771-5777.

Fang, Z.P., Mortimer, J.T. (1991) Selective activation of small motor axons by quasi-trapezoidal current pulses. IEEE Trans Biomed Eng 38(2):168-74.

Gorman, P.H., Mortimer, J.T. (1983) The effect of stimulus parameters on the recruitment characteristics of direct nerve stimulation. IEEE Trans Biomed Eng 30(7):407-414.

Grill, W.M. (2015) Model-based analyses and design of waveforms for efficient neural stimulation. Prog Brain Res 222: 147-162. doi: 10.1016/bs.pbr.2015.07.031. 
Groves, D. A., Brown, V. J. (2005). Vagal nerve stimulation: a review of its applications and potential mechanisms that mediate its clinical effects. Neurosci Biobehav Rev 29, 493500. doi: 10.1016/j.neubiorev.2005.01.004

Heck, C., Helmers, S. L., DeGiorgio, C. M. (2002). Vagus nerve stimulation therapy, epilepsy, and device parameters: scientific basis and recommendations for use. Neurology 59(6 Suppl. 4), S31-S37.

Huston, J. M., Gallowitsch-Puerta, M., Ochani, M., Ochani, K., Yuan, R., Rosas-Ballina, M., Ashok, M., Goldstein, R.S., Chavan, S.S., Pavlov, V.A., Metz, C.N., Yang, H., Czura, C.J., Wang, H., Tracey, K.J. (2007) Transcutaneous vagus nerve stimulation reduces serum high mobility group box 1 levels and improves survival in murine sepsis. Crit. Care Med. 35, 2762-2768.

Hutchins, A.P., Diez, D., Miranda-Saavedra D. (2013) The IL-10/STAT3-mediated anti inflammatory response: recent developments and future challenges Brief Funct Genomics 12:489-498.

Kalliolias, G.D., Ivashkiv, L.B. (2016) TNF biology, pathogenic mechanisms and emerging therapeutic strategies Nature Reviews Rheumatology 12: 49-62.

Khan S., Khan, S.A., Luo, X., Fattah, F.J., Saltarski, J., Gloria-McCutchen, Y., Lu, R., Xie, Y., Li Q., Wakeland, E., Gerber, D.E. (2019) Immune dysregulation in cancer patients developing immune-related adverse events. British Journal of Cancer 120(1):63-68. doi: 10.1038/s41416-018-0155-1.

Kong, S.S., Liu, J.J., Hwang, T.C., Yu, X.J., Zhao, M., Zhao, M., Yuan, B.X., Lu, Y., Kang, Y.M., Wang, B., Zang, W.J. (2012) Optimizing the parameters of vagus nerve stimulation by uniform design in rats with acute myocardial infarction, PLoS One 7(11):e42799. doi: 10.1371/journal.pone.0042799.

Koopman, F. A., S. S. Chavan, S. Miljko, S. Grazio, S. Sokolovic, P. R. Schuurman, A. D. Mehta, Y. A. Levine, M. Faltys, R. Zitnik, K. J. Tracey, and P. P. Tak. 2016. Vagus nerve stimulation inhibits cytokine production and attenuates disease severity in rheumatoid arthritis. Proc. Natl. Acad. Sci. U. S. A. 113: 8284-9.

Levine, Y. A., F. A. Koopman, M. Faltys, A. Caravaca, A. Bendele, R. Zitnik, M. J. Vervoordeldonk, and P. P. Tak. 2014. Neurostimulation of the Cholinergic Anti-Inflammatory Pathway Ameliorates Disease in Rat Collagen-Induced Arthritis. PLoS One 9: e104530.

Musselman, E.D., Pelot, N.A., Grill, W.M. (2019) Empirically based guideline for selecting vagus nerve stimulation parameters in epilepsy and heart failure. Cold Spring Harb Perspect Med. 9(7). pii: a034264. doi: 10.1101/cshperspect.a034264.

Olofsson, P.S., Levine, Y.A., Caracava, A., Chavan, S.S., Pavlov, V.A., Faltys, M., Tracey, K.J. (2015) Single-Pulse and Unidirectional Electrical Activation of the Cervical Vagus Nerve Reduces Tumor Necrosis Factor in Endotoxemia. Bioelectronic Medicine 2, 37-42. 
Steinberg, B.E., Silverman, H.A., Robbiati, S., Gunasekaran, M.K., Tsaava, T., Battinelli, E., Stiegler, A., Bouton, C.E., Chavan, S.S., Tracey, K.J., Huerta, P.T. (2016) Cytokinespecific neurograms in the sensory vagus nerve. Bioelectron Med 3:7-17.

Tracey, K.J. (2002) The inflammatory reflex. Nature. 420(6917):853-9.

Tsaava, T., Kressel, A.M., Uryu, K., Chavan, S.S., Tracey, K.J., Chang, E.H. (2019) Optogenetic activation of fiber-specific compound action potentials in the mouse vagus nerve. 2019 9th International IEEE/EMBS Conference on Neural Engineering (NER), 867-870 doi: 10.1109/NER.2019.8717050

Varzaneh, F.N., Keller, B., Unger, S., Aghamohammadi, A., Warnatz, K., Rezaei, N. (2014) Cytokines in Common Variable Immunodeficiency as Signs of Immune Dysregulation and Potential Therapeutic Targets - A Review of the Current Knowledge. J Clin Immunol 34: 524-543. doi: 10.1007/s10875-014-0053-0.

Watkins L.R., Goehler, L.E., Relton, J.K., Tartaglia, N., Silbert, L., Martin, D., Maier, S.F. (1995) Blockade of interleukin-1 induced hyperthermia by subdiaphragmatic vagotomy: Evidence for vagal mediation of immune-brain communication. Neurosci Lett 183(12):27-31

Yoo, P.B., Lio, H., Hincapie, J.G., Ruble, S.B., Hamann, J.J., Grill, W.M. (2016) Modulation of heart rate by temporally patterned vagus nerve stimulation in anesthetized dog. Physiol Rep 4(2): e12689.

Yoo, P.B., Lubock, N.B., Hincapie, J.G., Ruble, S.B., Hamann, J.J., Grill, W.M. (2013) Highresolution measurement of electrically-evoked vagus nerve activity in the anesthetized dog. J Neural Eng 10(2):026003. doi: 10.1088/1741-2560/10/2/026003.

Zanos, T.P., Silverman, H.A., Levy, T., Tsaava, T., Battinelli, E., Lorraine, P.W., Ashe, J.M., Chavan, S.S., Tracey, K.J., Bouton, C.B. (2018) Identification of cytokine-specific sensory neural signals by decoding murine vagus nerve activity Proceedings of the National Academy of Sciences 115: E4843-E4852.

\section{FUNDING}

This work was supported in part by DARPA (HR0011-15-2-0016) and NIH (1R35GM118182-01). 


\section{Figure 1}

A.
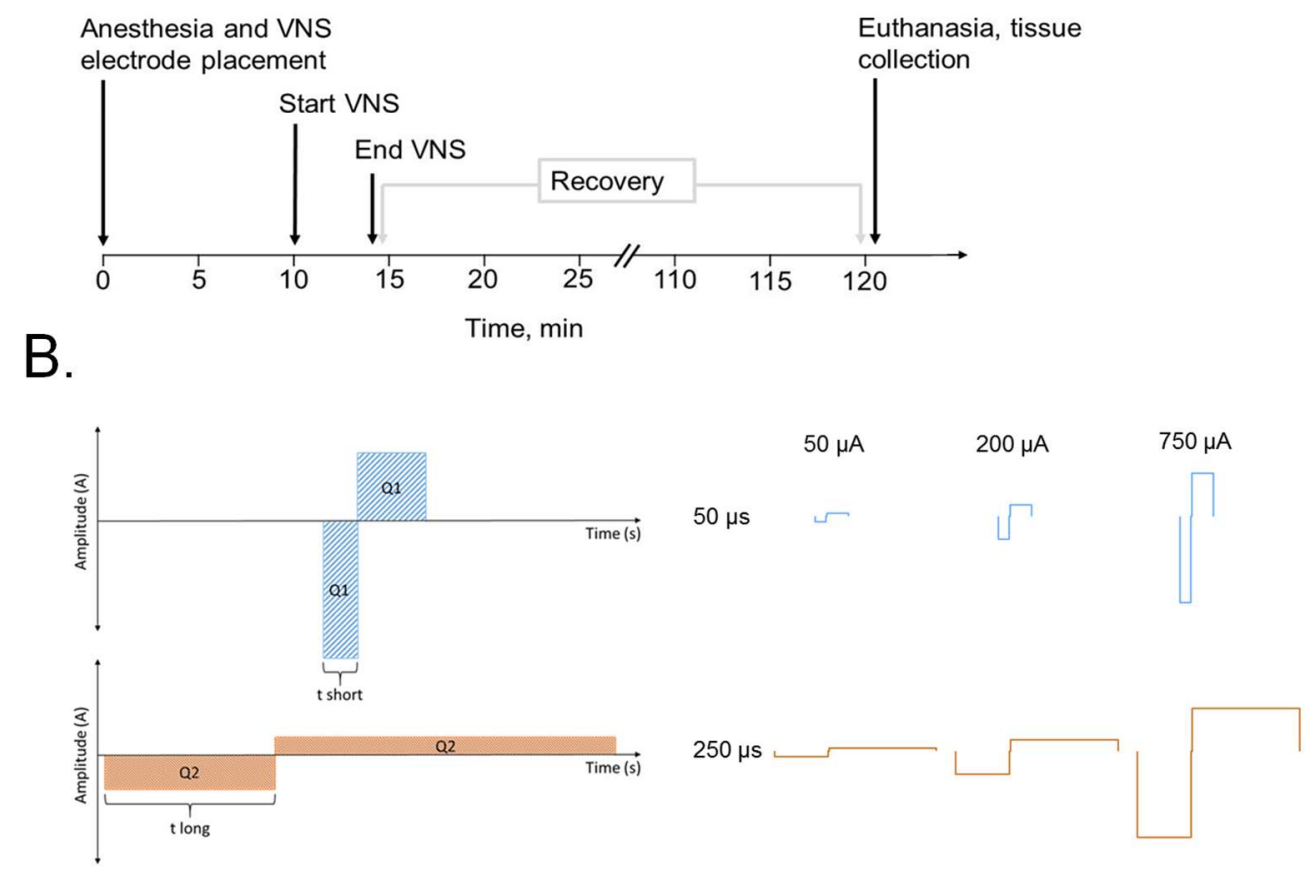

Figure 1. Experimental design and stimulation pulse waveforms. A.) Experimental timeline. Electrical stimulation pulse trains were applied to the exposed left cervical vagus nerve for four minutes under anesthesia. Following stimulation, the animals were recovered for two hours, euthanized, and whole blood was collected through cardiac puncture. B.) Schematic of the chargebalanced stimulation waveforms used during stimulation, with short pulse width (top) and long pulse width (bottom). The actual waveform shapes used in this study are shown to the right. 


\section{Figure 2}

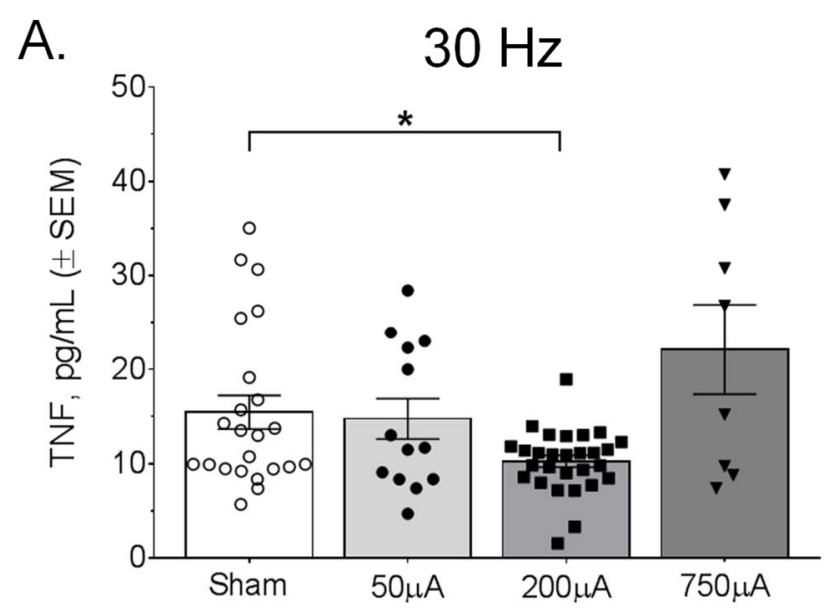

B.

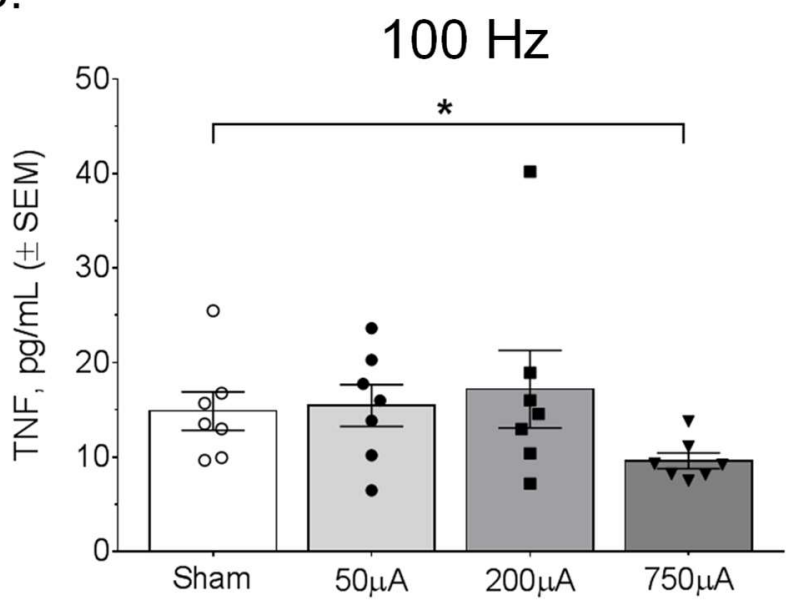

Figure 2. Specific stimulation amplitude and frequency combinations at $\mathbf{5 0} \mu \mathrm{s}$ pulse widths reduce serum TNFa levels. A.) A significant decrease in TNFa, compared to the sham group, was observed with $30 \mathrm{~Hz}$ stimulation and a pulse amplitude of $200 \mu \mathrm{A}$. B.) A significant decrease in TNFa was observed at $100 \mathrm{~Hz}$ stimulation with a pulse amplitude of $750 \mu \mathrm{A}$. 


\section{Figure 3}

A.

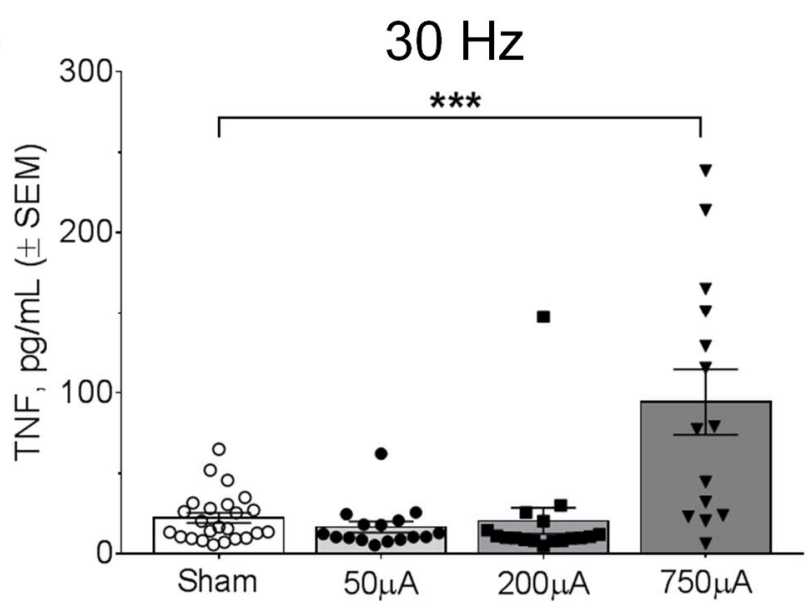

B.

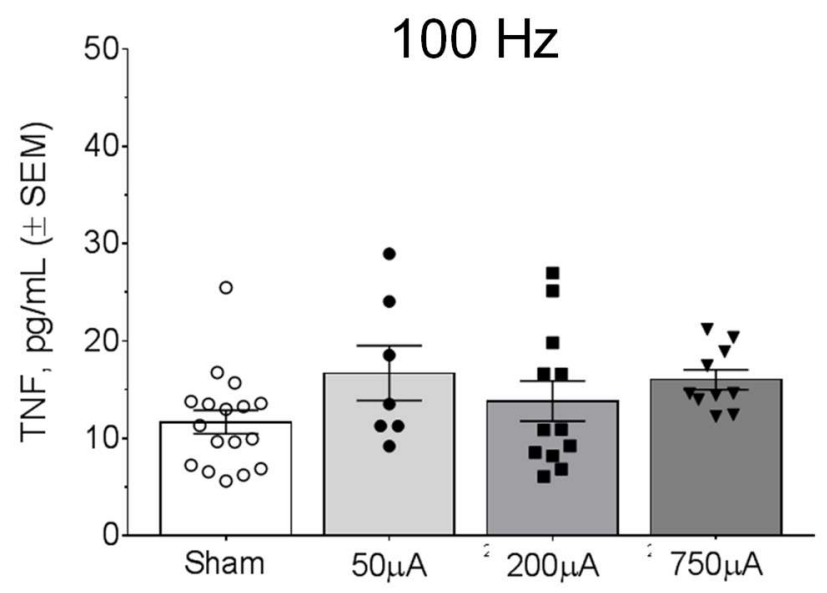

Figure 3. Serum TNFa is significantly increased by vagus nerve stimulation at $250 \mu \mathrm{s}$ for a specific parameter combination. A.) Stimulation resulted in a significant increase in TNF $\alpha$ at $30 \mathrm{~Hz}$ and 750 $\mu \mathrm{A}$ pulse amplitude, compared to the sham group. B.) No significant changes in serum TNFa were observed with the $250 \mu$ s pulse width at $100 \mathrm{~Hz}$. 


\section{Figure 4}

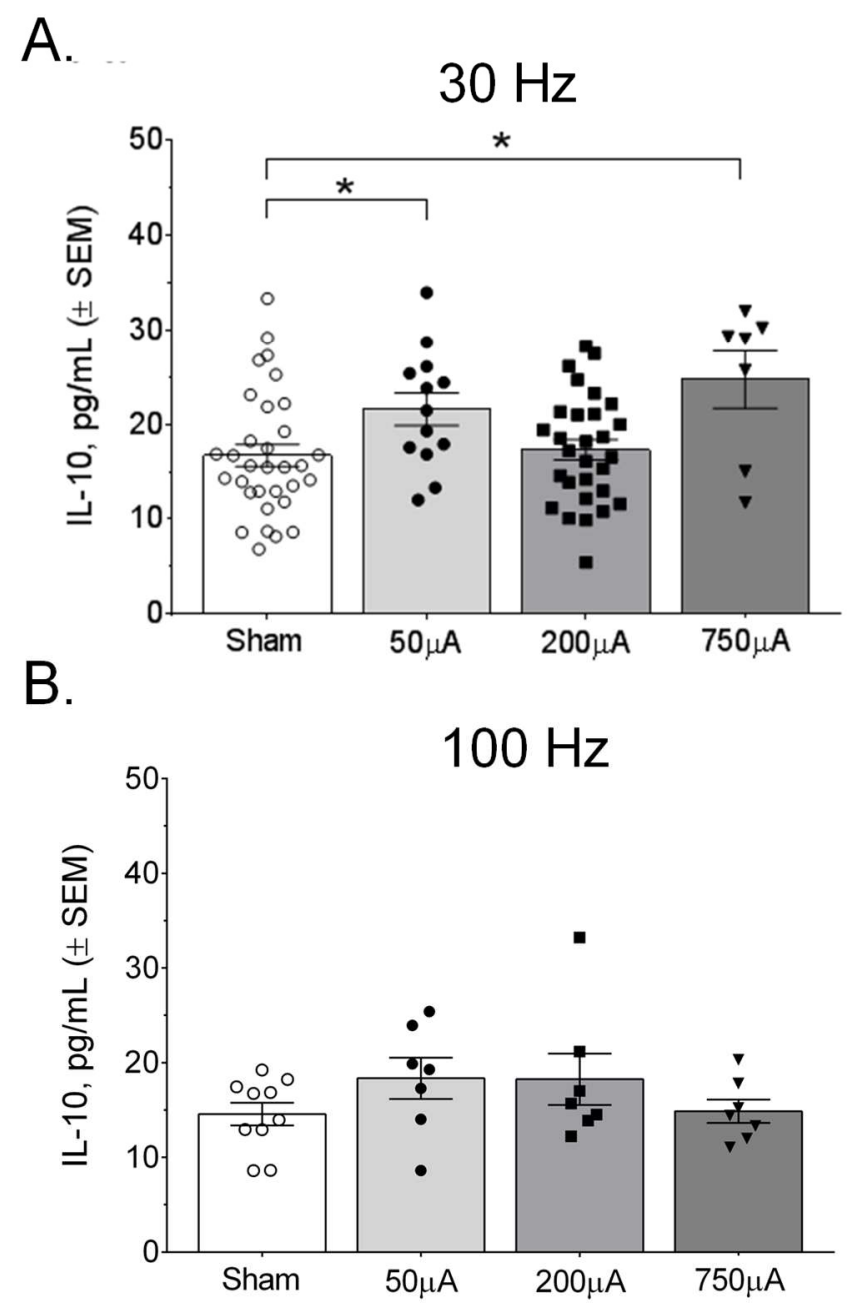

Figure 4. Serum IL-10 is increased by select parameters of electrical stimulation with $\mathbf{5 0} \mu \mathrm{s}$ pulse width. A.) Stimulation for $50 \mu \mathrm{s}$ at $30 \mathrm{~Hz}$ produced significant increases, compared to the sham group, in IL-10 for both $50 \mu \mathrm{A}$ and $750 \mu \mathrm{A}$ pulse amplitudes. B.) No changes in serum IL-10 were observed for $100 \mathrm{~Hz}$ stimulation across the four pulse amplitudes. 


\section{Figure 5}

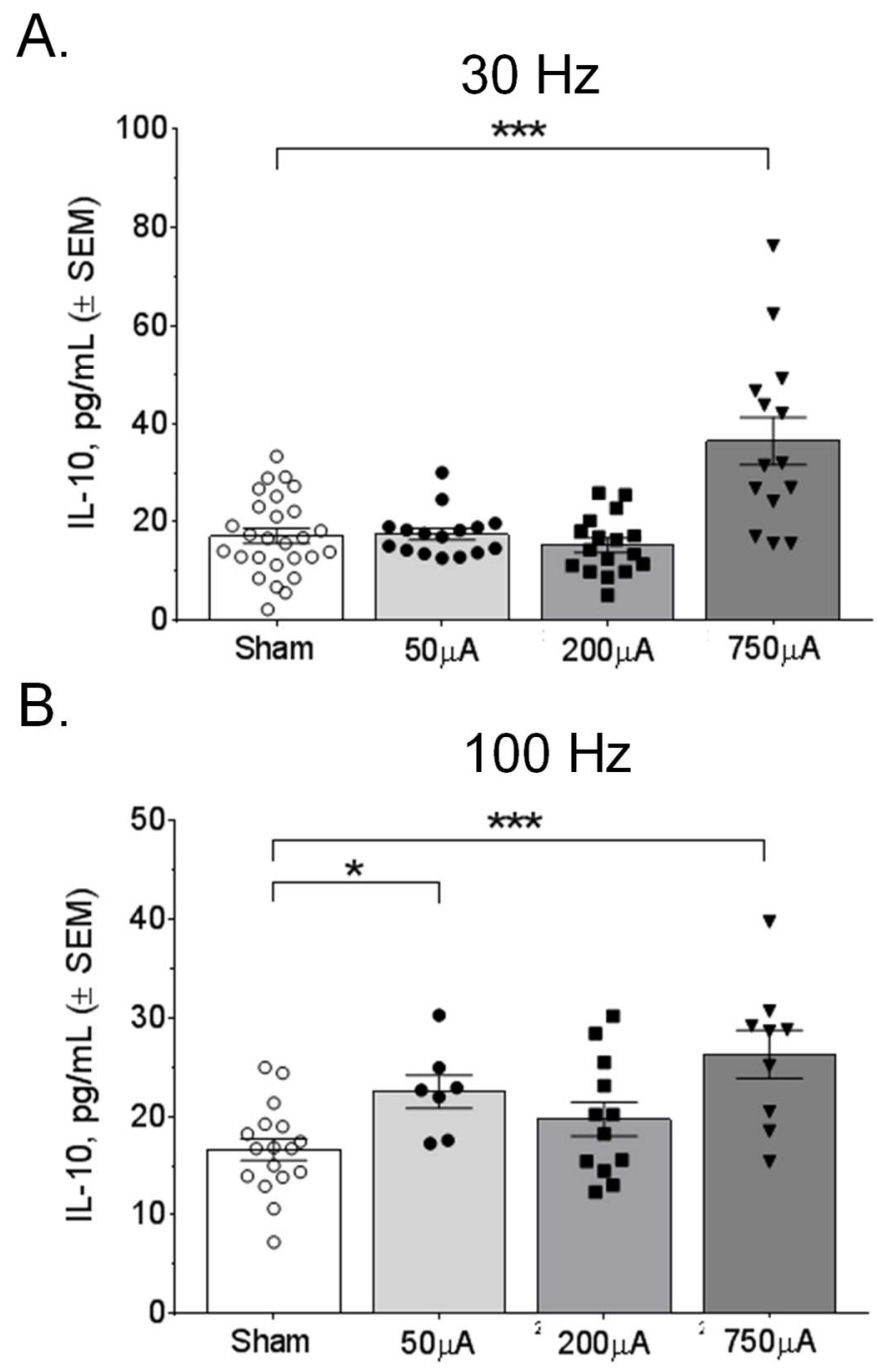

Figure 5. Nerve stimulation with $250 \mu s$ pulse width increased serum IL-10 at several different parameters. A.) Stimulation with $250 \mu \mathrm{s}$ pulses at $30 \mathrm{~Hz}$ produced a marked increase in IL-10 at the $750 \mu \mathrm{A}$ pulse amplitude. B.) Stimulation with $250 \mu \mathrm{s}$ pulses at $100 \mathrm{~Hz}$ produced significant increases at both $50 \mu \mathrm{A}$ and $750 \mu \mathrm{A}$ pulse amplitudes. 


\section{Figure 6}

A.

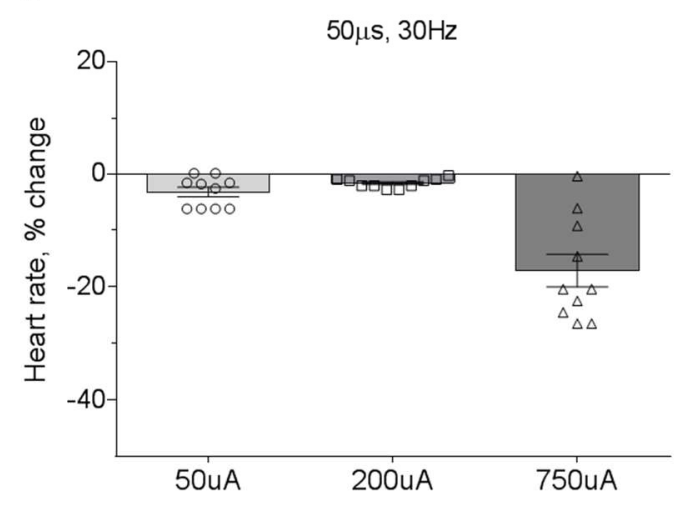

C.

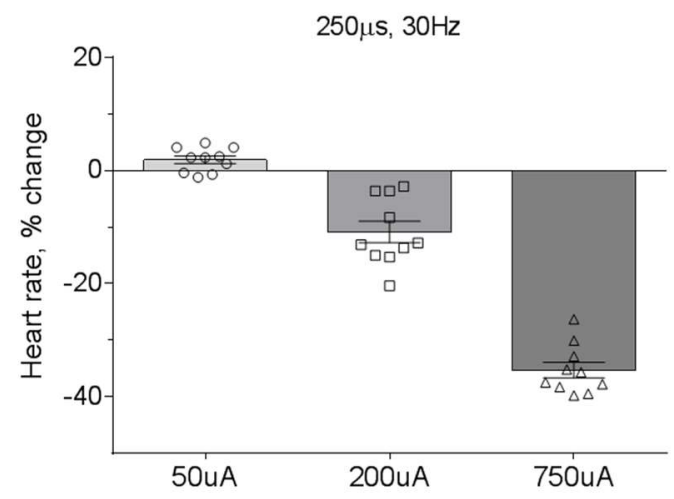

B.

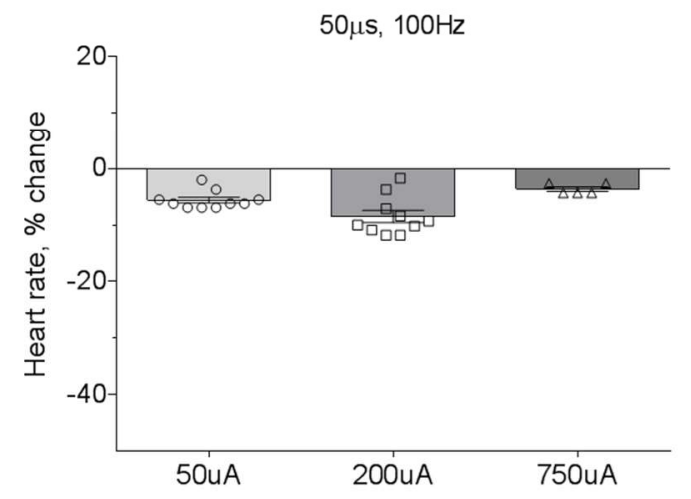

D.

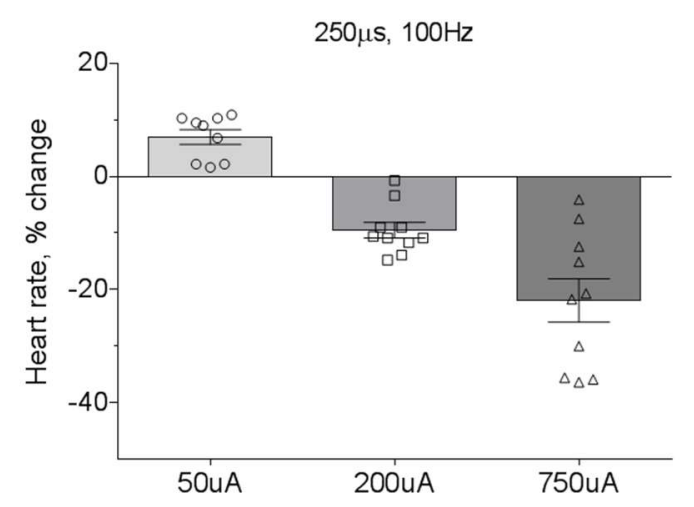

Figure 6. The effect of different vagus nerve stimulation parameters on heart rate. A.) Stimulation at $30 \mathrm{~Hz}$ with $50 \mu$ s pulse width resulted in bradycardia $(\geq 10 \%$ reduction in heart rate) at only the $750 \mu \mathrm{A}$ pulse amplitude. B.) At $100 \mathrm{~Hz}$, bradycardia was not observed with the $50 \mu$ s pulse width. C.) Stimulation with the longer $250 \mu \mathrm{s}$ pulse width resulted in bradycardia at 200 and $750 \mu \mathrm{A}$ pulse amplitudes. D.) The largest decrease in heart rate was observed with the $250 \mu$ s pulse width at $100 \mathrm{~Hz}$ and $750 \mu \mathrm{A}$ amplitude. 


\section{Table 1.}

Cytokine levels (mean \pm SEM) at $50 \mu$ s pulse

\begin{tabular}{|c|c|c|c|c|c|c|c|}
\hline Cytokine & Sham & $\begin{array}{l}50 \mathrm{uA} \\
30 \mathrm{~Hz}\end{array}$ & $\begin{array}{c}200 \mathrm{uA} \\
30 \mathrm{~Hz}\end{array}$ & $\begin{array}{c}750 \mathrm{uA} \\
30 \mathrm{~Hz}\end{array}$ & $\begin{array}{c}50 \mathrm{uA} \\
100 \mathrm{~Hz}\end{array}$ & $\begin{array}{l}200 \mathrm{uA} \\
100 \mathrm{~Hz}\end{array}$ & $\begin{array}{l}750 \mathrm{uA} \\
100 \mathrm{~Hz}\end{array}$ \\
\hline \multicolumn{8}{|l|}{ IFN-Y } \\
\hline & $0.64 \pm 0.15$ & $0.48 \pm 0.13$ & $0.34 \pm 0.05$ & $0.46 \pm 0.26$ & $0.39 \pm 0.11$ & $0.53 \pm 0.21$ & $0.20 \pm 0.02$ \\
\hline \multicolumn{8}{|l|}{ IL-12 p70 } \\
\hline & $37.27 \pm 5.72$ & $34.18 \pm 7.17$ & $47.35 \pm 6.77$ & $30.35 \pm 9.37$ & $41.24 \pm 11.16$ & $58.15 \pm 16.82$ & $43.77 \pm 10.46$ \\
\hline \multicolumn{8}{|l|}{ IL-1及 } \\
\hline & $1.79 \pm 0.32$ & $2.43 \pm 0.41$ * & $1.53 \pm 0.21$ & $2.03 \pm 0.38$ & $2.93 \pm 0.54$ & $2.15 \pm 0.52$ & $2.35 \pm 0.73$ \\
\hline \multicolumn{8}{|l|}{ IL-2 } \\
\hline & $1.75 \pm 0.70$ & $1.29 \pm 0.65$ & $1.82 \pm 0.71$ & $1.40 \pm 0.35$ & $2.25 \pm 0.64$ & $2.09 \pm 0.88$ & $0.36 \pm 0.10$ \\
\hline \multicolumn{8}{|l|}{ IL-4 } \\
\hline & $0.22 \pm 0.08$ & $0.37 \pm 0.16$ & $0.81 \pm 0.41$ & $0.47 \pm 0.28$ & $0.45 \pm 0.18$ & $0.43 \pm 0.10$ & $0.36 \pm 020$ \\
\hline \multicolumn{8}{|l|}{ IL-5 } \\
\hline & $3.12 \pm 0.34$ & $4.58 \pm 0.89$ & $4.02 \pm 0.75$ & $4.43 \pm 0.88$ & $5.38 \pm 1.05 *$ & $4.42 \pm 0.95$ & $1.85 \pm 0.20 *$ \\
\hline IL-6 & $\begin{array}{c}2289.58 \pm \\
331.97\end{array}$ & $\begin{array}{l}3270.73 \pm \\
323.83^{* *}\end{array}$ & $\begin{array}{c}2653.11 \pm \\
184.78 \text { * }\end{array}$ & $\begin{array}{l}5516.24 \pm \\
1139.26^{* *}\end{array}$ & $\begin{array}{c}2826.10 \pm \\
305.77\end{array}$ & $\begin{array}{c}2921.93 \pm \\
322.54 \text { * }\end{array}$ & $\begin{array}{c}2906.27 \pm \\
320.22 \text { * }\end{array}$ \\
\hline CXCL1 & $739.68 \pm 50.39$ & $\begin{array}{c}1004.54 \pm \\
86.69^{*}\end{array}$ & $\begin{array}{l}1074.40 \pm \\
60.44^{* * *}\end{array}$ & $\begin{array}{c}786.88 \pm \\
71.59\end{array}$ & $\begin{array}{l}1131.30 \pm \\
71.65^{* * *}\end{array}$ & $\begin{array}{c}1025.21 \pm \\
68.99^{* *}\end{array}$ & $\begin{array}{c}880.59 \pm \\
92.48\end{array}$ \\
\hline
\end{tabular}

Full multiplex panel results of serum cytokine levels following nerve stimulation with $50 \mu \mathrm{s}$ pulse width. Values in red indicate significant differences from the Sham group. ${ }^{*}, P<0.05$; ${ }^{* *}, P<0.01 ;{ }^{* * *} P<0.001$. 


\section{Table 2.}

Cytokine levels (mean \pm SEM) at $250 \mu$ s pulse

\begin{tabular}{|c|c|c|c|c|c|c|c|}
\hline Cytokine & Sham & $\begin{array}{l}50 \mathrm{uA} \\
30 \mathrm{~Hz}\end{array}$ & $\begin{array}{c}200 \mathrm{uA} \\
30 \mathrm{~Hz}\end{array}$ & $\begin{array}{c}750 \mathrm{uA} \\
30 \mathrm{~Hz}\end{array}$ & $\begin{array}{c}50 \mathrm{uA} \\
100 \mathrm{~Hz}\end{array}$ & $\begin{array}{l}200 \mathrm{uA} \\
100 \mathrm{~Hz}\end{array}$ & $\begin{array}{l}750 \mathrm{uA} \\
100 \mathrm{~Hz}\end{array}$ \\
\hline IFN-Y & $0.69 \pm 0.16$ & $0.58 \pm 0.22$ & $0.45 \pm 0.09$ & $0.96 \pm 0.48$ & $0.66 \pm 0.20$ & $0.51 \pm 0.12$ & $0.25 \pm 0.03$ \\
\hline IL-12 p70 & $50.31 \pm 15.11$ & $48.26 \pm 6.26$ & $48.83 \pm 13.70$ & $39.63 \pm 4.49$ & $52.80 \pm 13.92$ & $41.90 \pm 7.12$ & $78.17 \pm 9.33$ ** \\
\hline IL-1 $\beta$ & $3.10 \pm 0.91$ & $5.42 \pm 2.04$ ** & $2.01 \pm 0.45$ & $1.48 \pm 0.34$ & $4.07 \pm 0.52$ & $2.54 \pm 0.44$ & $2.15 \pm 0.29$ \\
\hline IL-2 & $2.85 \pm 1.12$ & $1.69 \pm 0.65$ & $3.05 \pm 1.23$ & $1.88 \pm 1.13$ & $5.21 \pm 2.31$ & $4.80 \pm 2.11$ & $2.63 \pm 0.56$ \\
\hline IL-4 & $0.34 \pm 0.08$ & $0.44 \pm 0.22$ & $0.38 \pm 0.17$ & $0.54 \pm 0.29$ & $0.67 \pm 0.42$ & $0.53 \pm 0.21$ & $0.20 \pm 0.06$ \\
\hline IL-5 & $2.97 \pm 0.36$ & $4.36 \pm 0.72$ & $5.48 \pm 1.26$ * & $6.13 \pm 1.41$ * & $9.50 \pm 2.74$ ** & $6.19 \pm 1.20$ *** & $3.55 \pm 0.65$ \\
\hline IL-6 & $\begin{array}{c}2125.23 \pm \\
243.76\end{array}$ & $\begin{array}{c}2981.44 \pm \\
376.14 \text { * }\end{array}$ & $\begin{array}{c}2911.30 \pm \\
405.83 \text { * }\end{array}$ & $\begin{array}{c}5880.09 \pm \\
1248.88^{* * *}\end{array}$ & $\begin{array}{l}3541.48 \pm \\
408.73^{* *}\end{array}$ & $\begin{array}{c}3040.72 \pm \\
394.06 \text { * }\end{array}$ & $\begin{array}{c}5490.44 \pm \\
473.28^{* * * *}\end{array}$ \\
\hline CXCL1 & $\begin{array}{c}798.88 \pm \\
55.16\end{array}$ & $\begin{array}{c}947.72 \pm \\
83.90\end{array}$ & $\begin{array}{c}1134.26 \pm \\
121.55 \text { * }\end{array}$ & $\begin{array}{c}907.32 \pm \\
114.89\end{array}$ & $\begin{array}{l}1192.14 \pm \\
80.46^{* * *}\end{array}$ & $\begin{array}{c}1717.80 \pm \\
170.34^{* \star \star *}\end{array}$ & $\begin{array}{c}1081.02 \pm \\
160.75\end{array}$ \\
\hline
\end{tabular}

Full multiplex panel results of serum cytokine levels following nerve stimulation with $250 \mu \mathrm{s}$ pulse width. Values in red indicate significant differences from the Sham group. ${ }^{*}, P<0.05$; ${ }^{* *}, P<0.01 ;{ }^{* *} P<0.001 ;{ }^{* * * *}, P<0.0001$. 